\title{
On the Hyperbolic Hausdorff Dimension of the Boundary of a Basin of Attraction for a Holomorphic Map and of Quasirepellers
}

by

\author{
Feliks PRZYTYCKI
}

Presented by Andrzej LASOTA

\begin{abstract}
Summary. We prove that the hyperbolic Hausdorff dimension of Fr $\Omega$, the boundary of the simply connected immediate basin of attraction $\Omega$ to an attracting periodic point of a rational mapping of the Riemann sphere, which is not a finite Blaschke product in some holomorphic coordinates, or a $2: 1$ factor of a Blaschke product, is larger than 1 . We prove a "local version" of this theorem, for a boundary repelling to the side of the domain.

The results extend an analogous fact for polynomials proved by A. Zdunik and relies on the theory elaborated by M. Urbański, A. Zdunik and the author in the late 80-ties. To prove that the dimension is larger than 1 , we use expanding repellers in $\partial \Omega$ constructed in $[\mathrm{P} 2]$.

To reach our results, we deal with a quasi-repeller, i.e. the limit set for a geometric coding tree, and prove that the hyperbolic Hausdorff dimension of the limit set is larger than the Hausdorff dimension of the projection via the tree of any Gibbs measure for a Hölder potential on the shift space, under a non-cohomology assumption. We also consider Gibbs measures for Hölder potentials on Julia sets.
\end{abstract}

0. Introduction. This paper completes [Z1], [Z2] and [P2]. In particular we prove the following

Theorem A. Let $f: \overline{\mathbb{C}} \rightarrow \overline{\mathbb{C}}$ be a rational map of degree $d \geq 2$ of the Riemann sphere and $\Omega$ be the simply connected immediate basin of attraction to a periodic attracting orbit. Then, provided $f$ is not a finite Blaschke product in some holomorphic coordinates, or a quotient of a Blaschke prod-

2000 Mathematics Subject Classification: Primary 37F35; Secondary 37F15, 37D25.

Key words and phrases: boundary of basin of attraction, Gibbs mesure, Hausdorff dimension, hyperbolic dimension, coding tree, iteration of holomorphic function, central limit theorem.

Supported by Polish KBN grant 2P03A 03425. 
uct by a rational function of degree 2, the hyperbolic Hausdorff dimension of $\operatorname{Fr} \Omega$ is larger than 1 .

The immediate basin of attraction to a periodic orbit $O=\left\{p_{1}, \ldots, p_{k-1}\right\}$ of period $k$ is the union of the components, intersecting $O$, of the set of points whose forward orbits converge to $O$. From now on we shall assume that $\Omega$ is the immediate basin of attraction to a fixed point, by considering $f^{k}$ in place of $f$ if $k>1$.

The hyperbolic Hausdorff dimension of an $f$-invariant set $K, \operatorname{HD}_{\text {hyp }}(K)$, is defined as the supremum of the Hausdorff dimensions of $f$-invariant subsets $X$ of $K$ such that $\left.f\right|_{X}$ is expanding, i.e. $\left|\left(\left.f^{N}\right|_{X}\right)^{\prime}\right|>1$ for an integer $N$. One can assume that the $X$ are isolated, i.e. there is an open set $U \supset X$ such that $\bigcap_{n \geq 0}\left(\left.f\right|_{U}\right)^{-n}(U)=X$. Moreover, in this definition we can consider only $X$ being Cantor sets.

In the case where $f$ is a polynomial and $\Omega$ is the basin of attraction to $\infty$, Theorem A was proved in [Z1]. In fact a stronger theorem was proved there: either a rational map $f$ has a parabolic orbifold (in particular $f(z)=z^{d}$ or a Chebyshev polynomial, if $f$ is a polynomial), or the Hausdorff dimension of the Julia set $J(f)$ (which is $\operatorname{Fr} \Omega$ for polynomials) is larger than the Hausdorff dimension of the measure $\mu_{\max }$ of maximal entropy. In the case of polynomials, $\mu_{\max }$ is equivalent to harmonic measure on $J(f)$ viewed from $\Omega$, whose Hausdorff dimension for $J(f)$ connected is equal to 1 [Mak].

To the best of our knowledge the question whether $\operatorname{HD}(J(f))>1$ is true for all rational $f$ not being Blaschke or a $2: 1$ factor of Blaschke and $J(f)$ connected, remains open, though a negative answer seems improbable. One can also ask about the Hausdorff dimension of non-one-point components of $J(f)$.

It is stated in [Z2, p. 635, end of Introduction] that Theorem A (in the RB version, see below) can be proved by the method of [Z1]. This is indeed true, an invariant expanding set $X$ of dimension larger than 1 can be constructed as in [Z1]. It is not however clear that $X \subset \mathrm{Fr} \Omega$; this needs some care in the construction. We verify it here using a more careful construction originated in $[\mathrm{PZ}]$ and carefully described in $[\mathrm{P} 2]$.

As in the other papers, we deal with another, local, version of Theorem A, that is, we prove

THEOREM A'. Assume that $f$ is defined and holomorphic on a neighbourhood $W$ of $\operatorname{Fr} \Omega$, where $\Omega$ is a simply connected domain in $\overline{\mathbb{C}}$ whose boundary has at least two points. Assume that $f(W \cap \Omega) \subset \Omega, f(\operatorname{Fr} \Omega) \subset \operatorname{Fr} \Omega$ and $\operatorname{Fr} \Omega$ repells to the side of $\Omega$, that is, $\bigcap_{n=0}^{\infty} f^{-n}(W \cap \operatorname{cl} \Omega)=\operatorname{Fr} \Omega$. Then either $\operatorname{HD}_{\text {hyp }}(\operatorname{Fr}(\Omega))>1$ or $\operatorname{Fr} \Omega$ is a real-analytic Jordan curve or arc.

Domains $\Omega$ with the properties as in Theorem $\mathrm{A}^{\prime}$ were introduced in [P1] and called $R B$-domains (repelling boundary to the side of $\Omega$ ). 
Theorem $\mathrm{A}^{\prime}$ yields Theorem A since if $f$ extends to a rational function on $\overline{\mathbb{C}}$, then $\operatorname{Fr} \Omega$ analytic implies that $f$ is special as in Theorem A by [Bro].

In Theorems $\mathrm{A}$ and $\mathrm{A}^{\prime}$ the proof of the dichotomy saying that either $f$ (or $\operatorname{Fr} \Omega$ ) is special or $\operatorname{HD}_{\text {hyp }}(\operatorname{Fr} \Omega)>1$ uses the fact that if $R: \mathbb{D} \rightarrow \Omega$ is a univalent holomorphic function from the unit disc to $\Omega$ (also called a Riemann mapping) then the radial limit $\widehat{R}$ exists $l$-almost everywhere, for $l$ the length measure (Fatou theorem). Denote by $g$ the extension to $\partial \mathbb{D}$ of the function $R^{-1} \circ f \circ R$ on $\mathbb{D}$. It is analytic and expanding (see [P1]). The function $\psi:=\log \left|g^{\prime}\right|-\log \left(\left|f^{\prime}\right| \circ \widehat{R}\right)$ composed with iterates of $g$, i.e. the sequence of random variables $\psi \circ g^{n}$, satisfies the central limit theorem (CLT) (see [PUZ]). This sequence is stationary with respect to a $g$-invariant measure $\nu$ on $\partial \mathbb{D}$ equivalent to $l$.

The case

$$
\sigma^{2}(\psi):=\lim _{n \rightarrow \infty} \frac{1}{n} \int\left(\sum_{k=0}^{n-1} \psi \circ g^{k}\right)^{2} d \nu=0
$$

leads to the real analyticity of $\operatorname{Fr} \Omega$ (see [Z2]), hence in the case $f$ is rational on $\overline{\mathbb{C}}$ to $f$ Blaschke or a $2: 1$ factor of Blaschke.

The case we need to consider is $\sigma^{2}>0$. Using deviations of the sums $\sum_{k=0}^{n-1} \psi \circ g^{k}$ from the expectation value $\int \psi d \nu=0$, we construct an iterated function system in $\operatorname{Fr} \Omega$ whose limit set has Hausdorff dimension larger than $\operatorname{HD}\left(\widehat{R}_{*}(\nu)\right)=1$ (the measure $\widehat{R}_{*}(\nu)$ is equivalent to the harmonic measure $\omega)$.

As in all the papers mentioned above it is enough to deal with a geometric coding tree version of Theorem A' (see Theorem B in Sections 1, 2). Namely we consider the Gibbs measure for an arbitrary Hölder continuous potential on the shift space, its projection $\mu$ via coding given by the tree to the limit set $\Lambda$, and prove that usually $\operatorname{HD}_{\text {hyp }}(\Lambda)>\operatorname{HD}(\mu)$. In Theorems $\mathrm{A}$ and $\mathrm{A}^{\prime}$, $\mu$ is equivalent to the harmonic measure. For a more general version see Theorem C in Section 3.

In Section 3, in Theorem $\mathrm{D}$, we consider $\mu$ given directly on the Julia set $J(f)$, as the Gibbs measure for a Hölder potential on $J(f)$, as in [DU], [P3] and [DPU].

1. Geometric coding trees. We recall the definitions from [PUZ], [PS], [PZ] and [P1]. Let $U$ be an open connected subset of the Riemann sphere $\overline{\mathbb{C}}$. Consider any holomorphic mapping $f: U \rightarrow \overline{\mathbb{C}}$ such that $f(U) \supset$ $U$ and $f: U \rightarrow f(U)$ is a proper map. Define $\operatorname{Crit}(f)=\left\{z: f^{\prime}(z)=0\right\}$, the set of critical points for $f$. Suppose that $\operatorname{Crit}(f)$ is finite. Consider any $z \in f(U)$. Let $z^{1}, \ldots, z^{d}$ be some $f$-preimages of $z$ in $U$, where $d \geq 2$. Consider smooth curves $\gamma^{j}:[0,1] \rightarrow f(U), j=1, \ldots, d$, joining $z$ to $z^{j}$ respectively (i.e. $\gamma^{j}(0)=z, \gamma^{j}(1)=z^{j}$ ), such that there are no critical 
values for iterates of $f$ in $\bigcup_{j=1}^{d} \gamma^{j}$, i.e. $\gamma^{j} \cap f^{n}(\operatorname{Crit}(f))=\emptyset$ for every $j$ and $n>0$.

Let $\Sigma^{d}:=\{1, \ldots, d\}^{\mathbb{Z}^{+}}$denote the one-sided shift space and $\sigma$ the shift to the left, i.e. $\sigma\left(\left(\alpha_{n}\right)\right)=\left(\alpha_{n+1}\right)$. For every sequence $\alpha=\left(\alpha_{n}\right)_{n=0}^{\infty} \in \Sigma^{d}$ we define $\gamma_{0}(\alpha):=\gamma^{\alpha_{0}}$. Suppose that for some $n \geq 0$, for every $0 \leq m \leq n$, and all $\alpha \in \Sigma^{d}$, the curves $\gamma_{m}(\alpha)$ are already defined. Suppose that for $1 \leq m \leq n$ we have $f \circ \gamma_{m}(\alpha)=\gamma_{m-1}(\sigma(\alpha))$, and $\gamma_{m}(\alpha)(0)=\gamma_{m-1}(\alpha)(1)$.

Define the curves $\gamma_{n+1}(\alpha)$ so that the previous equalities hold by taking suitable $f$-preimages of the curves $\gamma_{n}$. For every $\alpha \in \Sigma^{d}$ and $n \geq 0$ set $z_{n}(\alpha):=\gamma_{n}(\alpha)(1)$. Note that $z_{n}(\alpha)$ and $\gamma_{n}(\alpha)$ depend only on $\left(\alpha_{0}, \ldots, \alpha_{n}\right)$ so sometimes we consider $z_{n}$ and $\gamma_{n}$ as functions on blocks of symbols of length $n+1$. Sometimes it is convenient to denote $z$ by $z_{-1}$.

The graph $\mathcal{T}\left(z, \gamma^{1}, \ldots, \gamma^{d}\right)$ with the vertices $z$ and $z_{n}(\alpha)$ and edges $\gamma_{n}(\alpha)$ is called a geometric coding tree with the root at $z$. For every $\alpha \in \Sigma^{d}$ the subgraph composed of $z, z_{n}(\alpha)$ and $\gamma_{n}(\alpha)$ for all $n \geq 0$ is called a geometric branch and denoted by $b(\alpha)$.

For each $j=1, \ldots, d$ we define $f_{j}^{-1}$ on a small neighbourhood of $z$ as the branch of $f^{-1}$ mapping $z$ to $z^{j}$. For each $\alpha \in \Sigma^{d}$ the branch $f_{j}^{-1}$ has an analytic continuation $f_{j, \alpha}^{-1}$ along the curve $b(\alpha)$. Note that by the construction $f_{j, \alpha}^{-1}(b(\alpha))=b(j \alpha)$, where $j \alpha$ is the concatenation of the symbol $j$ and the sequence $\alpha$. By induction, for any block $v$ of $k$ symbols in $\{1, \ldots, d\}$, for $f_{v}^{-k}$ being the branch of $f^{-k}$ mapping $z$ to $z_{k-1}(v)$ and for $f_{v, \alpha}^{-k}$ being the analytic continuation along $b(\alpha)$, we get

$$
f_{v, \alpha}^{-k}(b(\alpha))=b(v \alpha)
$$

For infinite $\alpha$ the branch $b(\alpha)$ is called convergent if the sequence $\gamma_{n}(\alpha)$ is convergent to a point in $\operatorname{cl} U$. We define the coding map $z_{\infty}: \mathcal{D}\left(z_{\infty}\right) \rightarrow \operatorname{cl} U$ by $z_{\infty}(\alpha):=\lim _{n \rightarrow \infty} z_{n}(\alpha)$ on the domain $\mathcal{D}=\mathcal{D}\left(z_{\infty}\right)$ consisting of all $\alpha$ 's for which $b(\alpha)$ is convergent. It was proved in [PS] that $\mathcal{D}$ is the whole $\Sigma^{d}$ except a "thin" set. In particular for a Gibbs measure $\nu$ as in Theorem B below, $z_{\infty}(\alpha)$ exists for $\nu$-a.e. $\alpha$, hence the push forward measure $\left(z_{\infty}\right)_{*}(\nu)$ makes sense.

For each geometric branch $b(\alpha)$ denote by $b_{m}(\alpha)$ the part of $b(\alpha)$ starting from $z_{m}(\alpha)$, i.e. consisting of the vertices $z_{k}(\alpha), k \geq m$, and of the edges $\gamma_{k}(\alpha), k>m$.

If the map $f$ extends holomorphically to a neighbourhood of the closure of the limit set $\Lambda$ of a geometric coding tree, $\Lambda=z_{\infty}\left(\mathcal{D}\left(z_{\infty}\right)\right)$, then $\Lambda$ is called a quasi-repeller (see [PUZ]).

TheOREM B. Let $\Lambda$ be a quasi-repeller for a geometric coding tree $\mathcal{T}\left(z, \gamma^{1}, \ldots, \gamma^{d}\right)$ for a holomorphic map $f: U \rightarrow \overline{\mathbb{C}}$. Let $\nu$ be a $\sigma$-invariant Gibbs probability measure on $\Sigma^{d}$ for a Hölder continuous real-valued function 
$u$ on $\Sigma^{d}$. Let $\mu:=\left(z_{\infty}\right)_{*}(\nu)$. Assume that for $\psi:=-u-\operatorname{HD}(\mu) \log \left(\left|f^{\prime}\right| \circ z_{\infty}\right)$ the asymptotic variance $\sigma^{2}:=\sigma^{2}(\psi)$ is positive. Then there exists an $f$ invariant hyperbolic subset $X$ of $\Lambda$ such that $\operatorname{HD}(X)>\operatorname{HD}\left(\left(z_{\infty}\right)_{*}(\nu)\right)$.

Recall that a probability measure $\nu$ is called a Gibbs measure for a Hölder continuous function $u: \Sigma^{d} \rightarrow \mathbb{R}$ if there is a constant $C_{1}>0$ such that for every cylinder

$$
\mathcal{C}_{n-1}(\beta):=\left\{\alpha \in \Sigma^{d}: \alpha_{0}=\beta_{0}, \ldots, \alpha_{n-1}=\beta_{n-1}\right\}
$$

for a sequence $\beta=\left(\beta_{0}, \ldots, \beta_{n-1}\right) \in\{1, \ldots, d\}^{n}$, we have

$$
C_{1}^{-1} \leq \nu\left(\mathcal{C}_{n-1}\right) / \exp \left(S_{n}(u)(\alpha)-n P(\sigma, u)\right) \leq C_{1},
$$

where $P(\sigma, u)$ is the topological pressure for the shift mapping $\sigma$ and potential function $u$ (see [Bow] or [PU]) and $S_{n}(u)(\alpha)=\sum_{j=0}^{n-1} u \circ \sigma^{j}(x)$.

We can assume that $P(\sigma, u)=0$ by replacing $u$ by $u-P(\sigma, u)$. Then we have $\int \psi d \nu=0$ (see [PUZ, Sec. 4, Lemma 7]).

2. Proof of Theorem B. We proceed as in [P2]. Denote the natural extension of the one-sided shift $\sigma$ with the measure $\nu$ and Borel $\sigma$-algebra $\mathcal{F}$ by $\left(\widetilde{\Sigma^{d}}, \widetilde{\mathcal{F}}, \widetilde{\nu}\right)$. This is a two-sided shift space with the shift $\widetilde{\sigma}$ to the left. Denote the projection $\widetilde{\Sigma}^{d} \rightarrow \Sigma^{d}$ mapping $\alpha$ to $\left(\alpha_{0}, \alpha_{1}, \ldots\right)$ by $\pi_{+}$. For each $\alpha \in \widetilde{\Sigma}^{d}$ denote $\pi_{+}(\alpha)$ by $\alpha^{+}$.

The Lyapunov exponent $\chi(\mu):=\int \log \left|f^{\prime}\right| d \mu$ is positive, since $2 \chi(\mu) \geq$ $\mathrm{h}_{\mu}(f)=\mathrm{h}_{\nu}(\sigma)>0$ (cf. [P1] and [PUZ]).

By the existence of $z_{\infty} \nu$-a.e. and by Pesin theory (see [PZ, Lemma 1] for the version we apply), for every $\varepsilon>0$ we find a set $K \subset \widetilde{\Sigma}^{d}, \delta>0$, a positive integer $M$ and a constant $C>0$ such that $\widetilde{\nu}(K)>1-\varepsilon$ and for all $\alpha \in K$ and $n \geq 0$,

(i) $b_{M}\left(\alpha^{+}\right) \subset B\left(z_{\infty}\left(\alpha^{+}\right), \delta / 3\right)$,

(ii) there exist univalent branches $f_{\alpha}^{-n}$ of $f^{-n}$ on $B\left(z_{\infty}\left(\alpha^{+}\right), \delta\right)$ for all $n=1,2, \ldots$ mapping $z_{\infty}\left(\alpha^{+}\right)$to $z_{\infty}\left(\widetilde{\sigma}^{-n}(\alpha)^{+}\right)$, of distortion bounded by $C$, i.e.

$$
\sup _{x \in B\left(z_{\infty}\left(\alpha^{+}\right), \delta\right)}\left|\left(f_{\alpha}^{-n}\right)^{\prime}(x)\right| / \inf _{x \in B\left(z_{\infty}\left(\alpha^{+}\right), \delta\right)}\left|\left(f_{\alpha}^{-n}\right)^{\prime}(x)\right| \leq C .
$$

In the notation accompanying the property (1) these branches are the continuations along $b\left(\alpha^{+}\right)$of $f_{\left(\alpha_{-n}, \ldots, \alpha_{-1}\right)}^{-n}$, i.e. the branches $f_{\left(\alpha_{-n}, \ldots, \alpha_{-1}\right), \alpha^{+}}^{-n}$.

The assumption $\sigma^{2}(\psi)>0$ implies $\sigma^{2}\left(\psi \circ \pi_{+}, \widetilde{\sigma}^{-1}\right)=\sigma^{2}(\psi)>0$ for the asymptotic variance of the sequence of $\psi \circ \pi_{+}$composed with the iterates of the mapping $\widetilde{\sigma}^{-1}$. Due to CLT, verified in [PUZ], we know that there exists $\varepsilon_{1}>0$ such that for

$$
K_{n}:=\left\{\alpha \in \widetilde{\Sigma}^{d}: S_{n}(\psi)(\alpha) \geq \sqrt{n}\right\}
$$


we have

$$
\widetilde{\nu}\left(K_{n}\right) \geq \varepsilon_{1}
$$

for all $n$ large enough. Here $S_{n} \psi:=\sum_{j=0}^{n-1} \psi \circ \pi_{+} \circ \widetilde{\sigma}^{-j}$.

Consider $K_{n}^{\prime}:=K \cap K_{n}$.

For each $n$ large enough we choose a cylinder

$$
\mathcal{C}_{M}=\mathcal{C}_{M}(\beta):=\left\{\alpha \in \widetilde{\Sigma}^{d}: \alpha_{0}=\beta_{0}, \ldots, \alpha_{M}=\beta_{M}\right\}
$$

for a sequence $\beta=\left(\beta_{0}, \ldots, \beta_{M}\right) \in\{1, \ldots, d\}^{M+1}$, such that

$$
\widetilde{\nu}\left(\mathcal{C}_{M} \cap K_{n}^{\prime}\right) \geq \frac{\varepsilon_{1}}{2} \widetilde{\nu}\left(\mathcal{C}_{M}\right):=\xi,
$$

which is possible if $\varepsilon$ is small enough. Restricting to a subsequence $\mathcal{N}$ of $n$ 's we can fix one $\mathcal{C}_{M}$, in particular $\xi$ is fixed, independent of $n \in \mathcal{N}$.

Consider $L_{n}:=\widetilde{\sigma}^{-n}\left(\mathcal{C}_{M} \cap K_{n}^{\prime}\right)$.

By the Birkhoff ergodic theorem there exists $N \geq 0$ independent of $n$ such that for all $n \in \mathcal{N}$,

$$
\widetilde{\nu}\left(\left\{\alpha \in L_{n}: \exists i: 0 \leq i<N, \widetilde{\sigma}^{-i}(\alpha) \in K \cap \mathcal{C}_{M}\right\}\right) \geq \xi / 2 .
$$

Therefore for every $n \geq 0$ there exists $N^{\prime}$ with $0 \leq N^{\prime}<N$ such that, setting $n^{\prime}:=n+N^{\prime}$, for $A\left(n^{\prime}\right):=\left\{\alpha \in \mathcal{C}_{M} \cap K_{n}^{\prime}: \widetilde{\sigma}^{-n^{\prime}}(\alpha) \in K \cap \mathcal{C}_{M}\right\}$ we have

$$
\widetilde{\nu}\left(A\left(n^{\prime}\right)\right) \geq \xi / 2 N
$$

As in [P2] let $G_{n^{\prime}} \subset\{1, \ldots, d\}^{n^{\prime}}$ be the set of sequences $v=\left(v_{-n^{\prime}}, \ldots, v_{-1}\right)$ of length $n^{\prime}$ which are truncations of $\alpha \in A\left(n^{\prime}\right)$ to the coordinates $\left\{-n^{\prime}, \ldots,-1\right\}$. For each $v$ we choose one $\alpha=\alpha_{v}$ whose truncation is $v$.

We consider the iterated function system consisting of the branches $F_{v}:=$ $f_{v, \alpha^{+}}^{-n^{\prime}}$ on $B:=B\left(z_{M}(\beta), 2 \delta / 3\right)$, for $v \in G_{n^{\prime}}$. The branches do not depend on $\alpha^{+}$(see [P2]). It is proved in [P2] that indeed the $F_{v}$ map $B$ to $B$ and the limit set

$$
X^{\prime}:=\bigcap_{j \geq 0} \bigcup_{v^{1}, \ldots, v^{j} \in G_{n^{\prime}}} F_{v^{j}} \circ \cdots \circ F_{v^{1}}(\operatorname{cl} B)
$$

is in the quasi-repeller $\Lambda$. This set is invariant under $\Phi:=f^{n^{\prime}}$.

As in [Z1, Section 9] we estimate the topological pressure on $X^{\prime}$ for $t=\mathrm{HD}(\mu)$ using preimages of $y=z_{M}(\beta)$ (we could use any point in $B$ here), remembering that $C$ bounds distortion for all $F_{v}$ on $B$ and writing

$$
Q_{v, n, \alpha}(u):=u\left(v_{-n} \ldots v_{-1} \alpha^{+}\right)+\cdots+u\left(v_{-1} \alpha^{+}\right)
$$

as follows: 
(4)

$$
\begin{aligned}
& P\left(\Phi,-t \log \left|\Phi^{\prime}\right|\right) \\
= & \lim _{j \rightarrow \infty} \frac{1}{j} \log \sum_{v^{1}, \ldots, v^{j} \in G_{n^{\prime}}}\left|F_{v^{j}}^{\prime}\left(F_{v^{j-1}} \circ \cdots \circ F_{v^{1}}(y)\right)\right|^{t} \cdots\left|F_{v^{1}}^{\prime}(y)\right|^{t} \\
\geq & -t \log C \\
& +\log \sum_{v=\left(v_{-n^{\prime}}, \ldots, v_{-1}\right) \in G_{n^{\prime}}} \exp \left(\sqrt{n}+Q_{v, n, \alpha_{v}}(u)-\left(n^{\prime}-n\right) t \sup \log \left|f^{\prime}\right|\right) \\
\geq & -t \log \left(C \sup \left|f^{\prime}\right|^{N^{\prime}}\right)+\sqrt{n}+\log \sum_{v=\left(v_{\left.-n^{\prime}, \ldots, v_{-1}\right)} \exp Q_{v, n, \alpha_{v}}(u)\right.} \\
\geq & -t \log \left(C \sup \left|f^{\prime}\right|^{N^{\prime}}\right)+\sqrt{n}+C_{1}^{-1} \xi / 2 N,
\end{aligned}
$$

where the last estimate of the sum follows from (3) and from the definition of Gibbs measure (2).

Indeed, for each $v \in G_{n^{\prime}}$ we have

$$
Q_{v, n, \alpha_{v}}(u) \geq C_{1}^{-1} \widetilde{\nu}\left\{\alpha \in A\left(n^{\prime}\right): \alpha_{-n}=v_{-n}, \alpha_{-1}=v_{-1}\right\},
$$

which, summed over $v$, was estimated from below in (3).

The right hand side of (4) is positive for $n$ large enough. Hence $\operatorname{HD}(X)$ $>t$, as $\operatorname{HD}(X)$ is (the only) zero of $P\left(\Phi,-t \log \left|\Phi^{\prime}\right|\right)$.

Finally, we define an $f$-invariant isolated Cantor set in $\Lambda$ of Hausdorff dimension larger than $\mathrm{HD}(\mu)$ as in [P2] by setting

$$
X:=\bigcup\left\{f^{j}\left(X^{\prime}\right): j=0, \ldots, n^{\prime}-1\right\} .
$$

3. Generalizations. Gibbs measures for Hölder functions on $J(f)$. By the same considerations as in the proof of Theorem B one can prove the following

TheOREM C. Suppose that for a quasi-repeller $\Lambda$ as in Theorem $B, \nu$ is a $\sigma$-invariant probability measure on $\Sigma^{d}$, of positive entropy, and $u=$ $\log \left(\operatorname{Jac}_{\nu}\left(\sigma^{-1}\right)\right) \circ \sigma: \Sigma^{d} \rightarrow \mathbb{R}$, where $\operatorname{Jac}_{\nu}\left(\sigma^{-1}\right)$ is the Jacobian of $\sigma^{-1}$ with respect to $\nu$ (the branch of $\sigma^{-1}$ inverting $\sigma$ ). (We assume that $\sigma^{-1}$ is absolutely continuous with respect to $\nu$.) Suppose there exists $\varepsilon>0$ and $a$ sequence $a_{n} \rightarrow \infty$ such that for $K_{n}:=\left\{\alpha \in \widetilde{\Sigma}^{d}: S_{n}(\psi)(\alpha) \geq a_{n}\right\}$ with $S_{n}(\psi)$ being the "backward sum" defined in the proof of Theorem $\mathrm{B}$, we have $\widetilde{\nu}\left(K_{n}\right) \geq \varepsilon$. Then there exists an $f$-invariant hyperbolic subset $X$ of $\Lambda$ such that $\operatorname{HD}(X)>\operatorname{HD}\left(\left(z_{\infty}\right)_{*}(\nu)\right)$.

To prove Theorem $\mathrm{C}$ we choose, for each $v$ in (5), $\alpha_{v} \in A\left(n^{\prime}\right)$ such that for $B_{v}:=\left\{v \alpha^{+} \in \Sigma^{d}: \alpha \in A\left(n^{\prime}\right), \alpha_{j}=v_{j} \forall j=-n, \ldots,-1\right\}$,

$$
\exp Q_{v, n, \alpha_{v}}(u) \geq \int_{B_{v}} \exp Q_{v, n, \alpha}(u) d\left(\nu \circ \sigma^{n}\right)\left(v \alpha^{+}\right)=\nu\left(B_{v}\right)
$$

and next, as in Section 2, we use $\sum \nu\left(B_{v}\right)=\widetilde{\nu}\left(A\left(n^{\prime}\right)\right) \geq$ const $>0$. 
We do not need to assume Hölder continuity of $u$.

We can apply Theorem $\mathrm{C}$ to a class of measures discussed in the following

TheOREM D. Let $f$ be a rational mapping of degree $d \geq 2$ on the Riemann sphere. Let $\mu$ be the Gibbs measure for a Hölder function $\varphi: J(f) \rightarrow \mathbb{R}$ such that the pressure satisfies $P(f, \varphi)>\sup \varphi$ (see [P3] and [DU]). Then, if $\varphi$ is not cohomologous to $-\mathrm{HD}(\mu) \log \left|f^{\prime}\right|$, we get

$$
\operatorname{HD}(\mu)<\operatorname{HD}_{\text {hyp }}(J(f)) .
$$

Proof. Theorem D follows from Theorem $\mathrm{C}$ under the additional assumption that there exists a geometric coding tree $\mathcal{T}\left(z, \gamma^{1}, \ldots, \gamma^{d}\right)$, with $d$ being the degree of $f$, and there exists a probability measure $\nu$ on $\Sigma^{d}$ such that

$$
\mu=\left(z_{\infty}\right) *(\nu)
$$

This holds for example if $\operatorname{diam}\left(\gamma_{n}(\alpha)\right) \rightarrow 0$ as $n \rightarrow \infty$, uniformly over $\alpha$ (see $[\mathrm{P} 4]$ ), in particular if $z$ is taken in the basin of attraction to $\infty$ for $f$ a polynomial.

We do not know whether $\mu=\left(z_{\infty}\right)_{*}(\nu)$ in the general situation for every $\mu$ of positive Lyapunov exponent.

Let us assume that $(*)$ holds. Since in view of the Variational Principle (see [P3] or [DU]), $\mathrm{h}_{\mu}(f)>0$ because $P(f, \varphi)>\sup \varphi$, we get $\mathrm{h}_{\nu}(f)>0$.

Now, to apply Theorem $\mathrm{C}$, it is sufficient to prove that $\psi=-\varphi-$ $\operatorname{HD}(\mu) \log \left|f^{\prime}\right|$ satisfies some assumptions sufficient for CLT to hold. Namely, it is sufficient to prove that

$$
\sum_{n=0}^{\infty}\left\|\mathcal{L}^{n}(\psi / h)-\mu(\psi / h)\right\|_{2}<\infty
$$

(see [DPU] and [Gor]). Here the norm is in $L_{2}(\mu)$ (square integrable functions) and $\mathcal{L}$ is the normalized transfer operator, namely the transfer operator for the function $\varphi_{0}:=\varphi-P(f, \varphi)+h \circ f-h$, where $h$ is the eigenfunction for the transfer operator with potential $\varphi$ corresponding to the leading eigenvalue equal to $\exp P(f, \varphi)$. By [DPU], $h$ is Hölder.

By [DPU], $\mathcal{L}^{n}(\varphi / h)-\mu(\varphi / h)=O(\exp (-\sqrt{n}))$, even in $L_{\infty}$. (N. Haydn [Hay] proved that the rate of convergence is in fact exponential.)

So it is sufficient to discuss $\left\|\mathcal{L}^{n}\left(\left(\log \left|f^{\prime}\right|\right) / h\right)-\mu\left(\left(\log \left|f^{\prime}\right|\right) / h\right)\right\|_{2}$.

Note that for any $\eta \in L_{2}(\mu)$, using $\sum_{y \in f^{-1}(x)} \exp \varphi_{0}(y) \equiv 1$ and the convexity of the square function, we get

$$
\begin{aligned}
& \|\mathcal{L}(\eta)\|_{2}^{2}=\int|\mathcal{L}(\eta)|^{2} d \mu=\int\left|\sum_{y \in f^{-1}(x)}\left(\exp \varphi_{0}(y)\right) \eta(y)\right|^{2} d \mu(x) \\
& \quad \leq \int \sum_{y \in f^{-1}(x)}\left(\exp \varphi_{0}(y)\right)|\eta|^{2}(y) d \mu(x) \leq \int \mathcal{L}\left(|\eta|^{2}\right) d \mu=\int|\eta|^{2} d \mu=\|\eta\|_{2}^{2}
\end{aligned}
$$


We shall use also the following fact (see [DPU, (3.1)]): For every $0<$ $\alpha \leq 1$ there exists $\tau>0$ such that

$$
\sup _{n \geq 0}\left\|\mathcal{L}^{n}(\eta)\right\|_{\mathcal{H}_{\tau}}<\infty \quad \forall \eta \in \mathcal{H}_{\alpha}
$$

where $\|\cdot\|_{\mathcal{H}_{a}}$ denotes the Hölder norm in the Banach space $\mathcal{H}_{a}$ of real-valued Hölder continuous functions on $J(f)$ (Lipschitz for $a=1$ ).

The constant $\tau$ has been chosen in [DPU] so that the operators $\mathcal{L}^{n}$ are continuous. So, by the Banach-Steinhaus theorem (or direct analysis of the proof of the above estimate in [DPU]), there is a common bound $A$ for the norms of the family of the operators $\mathcal{L}^{n}: \mathcal{H}_{\alpha} \rightarrow \mathcal{H}_{\tau}$.

Having this, we can apply Lemma 1 below (Lemma 4.4 from [DPU]) for functions of the form $\eta=\mathcal{L}^{k}(\xi), k \geq 0$, with $C_{1}$ depending only on $\|\xi\|_{\mathcal{H}_{\alpha}}$.

LEMma 1. There exist $0<\lambda<1,0<\gamma<1, C>0$ such that for every $C_{1}>0$ and $\Theta>0$, and for $n=n(\Theta)$ being the least positive integer greater than $\left(\log \Theta-\log C C_{1}\right) / \log \lambda$, if $\mu(\eta)=0,\|\eta\|_{\mathcal{H}_{\tau}}<C_{1}$ and $\|\eta\|_{\infty}<\Theta$, then

$$
\left\|\mathcal{L}^{n}(\eta)\right\|_{\infty}<\gamma \Theta
$$

Now we can prove (6). In what follows, $C$ will denote various positive constants, depending only on $f$.

Let $\eta:=-\left(\log \left|f^{\prime}\right|\right) / h-\mu\left(-\left(\log \left|f^{\prime}\right|\right) / h\right)$ and $\eta_{n}:=\min (\eta, \sqrt{n})$.

Then, summing over $c \in \operatorname{Crit}(f) \cap J(f)$, using $\mu(B(z, r)) \leq r^{\kappa}$ for a constant $\kappa>0$ and for all discs $B(z, r)$ small enough (which follows as in the proof of Lemma 4 in [PUZ]), for all $n$ large enough we get

$$
\begin{aligned}
\left\|\eta-\eta_{n}\right\|_{2}^{2} & \leq \sum_{c} \sum_{j=C^{-1} n}^{\infty} C(\sqrt{j})^{2} \mu(B(c, \exp (-\sqrt{j}))) \\
& \leq \sum_{c} \sum_{j=C^{-1} n}^{\infty} C j \exp (-\kappa \sqrt{j}) \leq \exp \left(-(\kappa / 2) \sqrt{C^{-1} n}\right) .
\end{aligned}
$$

$\left(C^{-1}\right.$ in the summation ranges also depends on inf $h$, i.e. on $\varphi$.)

Hence, with the use of (7), we get

$$
\left\|\mathcal{L}^{n}\left(\eta-\eta_{n}\right)-\mu\left(\eta-\eta_{n}\right)\right\|_{2}^{2} \leq \exp \left(-(\kappa / 2) \sqrt{C^{-1} n}\right) .
$$

Now, let us consider $\eta_{n}$. Its Lipschitz norm, and hence its $\alpha$-Hölder norm, is bounded by

$$
C(\sqrt{n+1}-\sqrt{n}) /(\exp (-\sqrt{n})-\exp (-\sqrt{n+1})) \leq C \exp \sqrt{n} .
$$

Hence $\left\|\mathcal{L}^{n}\left(\eta_{n}\right)-\mu\left(\eta_{n}\right)\right\|_{\mathcal{H}_{\tau}} \leq A C \exp \sqrt{n}$.

Let us iterate Lemma $1 s$ times starting from $\xi_{n}:=\eta_{n}-\mu\left(\eta_{n}\right)$. We get, for $\Theta:=\left\|\xi_{n}\right\|_{\infty} \leq 2 \sqrt{n}$ and all $s$,

$$
\left\|\mathcal{L}^{N(s)}\left(\xi_{n}\right)\right\|_{\infty} \leq 2 \gamma^{s} \sqrt{n},
$$


where $N(s):=n(\Theta)+n(\gamma \Theta)+\cdots+n\left(\gamma^{s-1} \Theta\right)$. We have

$$
\begin{aligned}
N(s) & \leq \sum_{j=0}^{s-1}\left(\log \left(\gamma^{j} 2 \sqrt{n}\right)-\log A C-\sqrt{n}\right) / \log \lambda \\
& \leq \frac{s(s-1)}{2} \frac{\log \gamma}{\log \lambda}+s \frac{\log n}{\log (1 / \lambda)}+s \frac{\log A C}{\log (1 / \lambda)}+s \frac{\sqrt{n}}{\log (1 / \lambda)} .
\end{aligned}
$$

If we want $N \leq n$, it is sufficient that the right hand side in (8) is less than $n$, which holds if $s$ satisfies

$$
A_{1} s^{2}+A_{2} s \log n+A_{3} s+A_{4} s \sqrt{n} \leq n
$$

for positive constants $A_{1}, A_{2}, A_{3}, A_{4}$ resulting from (8), i.e. if $s \leq C \sqrt{n}$. Then

$$
\left\|\mathcal{L}^{n}\left(\xi_{n}\right)\right\|_{\infty} \leq 2 \gamma^{C \sqrt{n}} \leq \exp (-\vartheta \sqrt{n}) .
$$

for $\vartheta=-(C / 2) \log \gamma$. Thus we proved the convergence of the series in (6). Moreover the sequence summed in $(6)$ is $O(\exp (-\vartheta \sqrt{n}))$ for a constant $\vartheta>0$. Hence, setting $\psi_{0}:=\psi / h-\mu(\psi / h)$, we get

$$
\sum_{n=1}^{\infty} n \int \psi_{0}\left(\psi_{0} \circ f^{n}\right) d \mu=\sum_{n=1}^{\infty} n \int \mathcal{L}^{n}\left(\psi_{0}\right) \psi_{0} d \mu \leq \sum_{n=1}^{\infty} n\left\|\mathcal{L}^{n}\left(\psi_{0}\right)\right\|_{2}\left\|\psi_{0}\right\|_{2}<\infty .
$$

So by the assumption that $\psi$ is not cohomologous to 0 , we get $\sigma^{2}(\psi / h)>0$ (see [PUZ, Lemma 1]).

If we do not assume $(*)$ we cannot use Theorem C. Fortunately, in the case we look for a hyperbolic $X$ in $J(f)$ rather than in a proper subset of $J(f)$, lifting the problem to the shift space is purely formal. So we can use a counterpart of Theorem $\mathrm{C}$ directly on $J(f)$, for $u$ being the logarithm of the Jacobian of $f^{-1}$ with respect to $\mu$ on $J(f)$ of positive Lyapunov exponent, for $\psi:=-u-\operatorname{HD}(\mu) \log \left|f^{\prime}\right|$. This ends the proof of Theorem D.

ConjeCture 1 . For any rational map $f$ there is at most one $f$-invariant probability measure $\mu$ on the Julia set $J(f)$, of positive Lyapunov exponent, such that $\mathrm{HD}(\mu)=\operatorname{HD}_{\text {hyp }}(J(f))$.

In other words, Conjecture 1 says that there is at most one measure $\mu$ which is an equilibrium measure for the potential function

$$
\beta:=-\operatorname{HD}_{\text {hyp }}(J(f)) \log \left|f^{\prime}\right| \text {. }
$$

Indeed, $\operatorname{HD}(\mu)=\mathrm{h}_{\mu}(f) / \chi(\mu)$ (see $[\mathrm{Ma}]$ ), hence $\mathrm{h}_{\mu}(f)-\operatorname{HD}(\mu) \chi(\mu)=0$. Since $P\left(\left.f\right|_{J(f)}, \beta\right)=0$ (see [P5] or [PRS]), $\mu$ is an equilibrium measure for $\beta$ iff $\operatorname{HD}(\mu)=\operatorname{HD}_{\text {hyp }}(J(f))$.

A stronger conjecture would be if we omit the positive Lyapunov exponent assumption.

Let us note that such a measure $\mu$ exists (and is indeed unique) for $f$ topological Collet-Eckmann (see $[\mathrm{PR}])$. In this case $\operatorname{HD}_{\text {hyp }}(J(f))=\mathrm{HD}(J(f))$ 
(see [P5]), and $\operatorname{Jac}_{\mu}(f)$ is cohomologous in measurable functions to $\operatorname{HD}(J(f))$ $\cdot \log \left|f^{\prime}\right|$ on $J(f)$. The function giving the cohomology is $\log d \mu / d m$, where $m$ is a unique conformal measure with exponent $\operatorname{HD}_{\text {hyp }}(J(f))$ (see $[\mathrm{PR}]$ ).

Acknowledgements. We wish to thank Juan Rivera-Letelier for several useful remarks.

\section{References}

[Bow] R. Bowen, Equilibrium States and the Ergodic Theory of Anosov Diffeomorphisms, Lecture Notes in Math. 470, Springer, Berlin 1975.

[Bro] H. Brolin, Invariant sets under iteration of rational functions, Ark. Mat. 6 (1965), 103-144.

[DPU] M. Denker, F. Przytycki and M. Urbański, On the transfer operator for rational functions on the Riemann sphere, Ergodic Theory Dynam. Systems 16 (1996), 255-266.

[DU] M. Denker and M. Urbański, Ergodic theory of equilibrium states for rational maps, Nonlinearity 4 (1991), 103-134.

[Gor] M. I. Gordin, The central limit theorem for stationary processes, Dokl. Akad. Nauk SSSR 188 (1969), 739-741 (in Russian); English transl.: Soviet Math. Dokl. 10 (1969), 1174-1176.

[Hay] N. Haydn, Convergence of the transfer operator for rational maps, Ergodic Theory Dynam. Systems 19 (1999), 657-669.

[Mak] N. Makarov, On the distortion of boundary sets under conformal mappings, Proc. London Math. Soc. 51 (1985), 369-384.

[Ma] R. Mañé, The Hausdorff dimension of invariant probabilities of rational maps, in: Dynamical Systems (Valparaiso, 1986), Lecture Notes in Math. 1331, Springer, 1988, 86-117.

[P1] F. Przytycki, Riemann map and holomorphic dynamics, Invent. Math. 85 (1986), 439-455.

[P2] -, Expanding repellers in limit sets for iteration of holomorphic functions, Fund. Math. 186 (2005), 85-96.

[P3] -, On the Perron-Frobenius-Ruelle operator for rational maps on the Riemann sphere and for Hölder continuous functions, Bol. Soc. Brasil. Mat. 20 (1990), 95-125.

[P4] - Accessibility of typical points for invariant measures of positive Lyapunov exponents for iterations of holomorphic maps, Fund. Math. 144 (1994), 259-278.

[P5] - Conical limit sets and Poincaré exponent for iterations of rational functions, Trans. Amer. Math. Soc. 351 (1999), 2081-2099.

[PR] F. Przytycki and J. Rivera-Letelier, Statistical properties of topological ColletEckmann maps, submitted.

[PRS] F. Przytycki, J. Rivera-Letelier and S. Smirnov, Equality of pressures for rational functions, Ergodic Theory Dynam. Systems 23 (2004), 891-914.

[PS] F. Przytycki and J. Skrzypczak, Convergence and pre-images of limit points for coding trees for iterations of holomorphic maps, Math. Ann. 290 (1991), 425-440.

[PU] F. Przytycki and M. Urbański, Fractals in the Plane, Ergodic Theory Methods, Cambridge Univ. Press, to appear. Available at http://www.math.unt.edu/ ${ }^{\sim}$ urbanski and http://www.impan.gov.pl/ ${ }^{\sim}$ feliksp. 
[PUZ] F. Przytycki, M. Urbański and A. Zdunik, Harmonic, Gibbs and Hausdorff measures for holomorphic maps, Part 1, Ann. of Math. 130 (1989), 1-40, Part 2, Studia Math. 97 (1991), 189-225.

[PZ] F. Przytycki and A. Zdunik, Density of periodic sources in the boundary of a basin of attraction for iteration of holomorphic maps: geometric coding trees technique, Fund. Math. 145 (1994), 65-77.

[Z1] A. Zdunik, Parabolic orbifolds and the dimension of the maximal measure for rational maps, Invent. Math. 99 (1990), 627-649.

[Z2] - Harmonic measure versus Hausdorff measures on repellers for holomorphic maps, Trans. Amer. Math. Soc. 326 (1991), 633-652.

Feliks Przytycki

Institute of Mathematics

Polish Academy of Sciences

00-956 Warszawa, Poland

E-mail: feliksp@impan.gov.pl

Received January 23, 2006;

received in final form May 24, 2006 